\title{
Characterisation of Systems for Raman-Assisted High-Speed Wavelength Conversion
}

\author{
Galili, Michael; Oxenløwe, Leif Katsuo; Zibar, Darko; Clausen, Anders; Jeppesen, Palle
}

Published in:

Conference on Lasers and Electro-Optics, 2005. CLEO

Link to article, DOI:

10.1109/CLEO.2005.201754

Publication date:

2005

Document Version

Publisher's PDF, also known as Version of record

Link back to DTU Orbit

Citation (APA):

Galili, M., Oxenløwe, L. K., Zibar, D., Clausen, A., \& Jeppesen, P. (2005). Characterisation of Systems for Raman-Assisted High-Speed Wavelength Conversion. In Conference on Lasers and Electro-Optics, 2005. CLEO (pp. CMQ2). IEEE. https://doi.org/10.1109/CLEO.2005.201754

\section{General rights}

Copyright and moral rights for the publications made accessible in the public portal are retained by the authors and/or other copyright owners and it is a condition of accessing publications that users recognise and abide by the legal requirements associated with these rights.

- Users may download and print one copy of any publication from the public portal for the purpose of private study or research.

- You may not further distribute the material or use it for any profit-making activity or commercial gain

- You may freely distribute the URL identifying the publication in the public portal 


\title{
Characterisation of Systems for Raman-Assisted High- Speed Wavelength Conversion
}

\author{
M. Galili, L.K. Oxenløwe, D. Zibar, A.T. Clausen and P. Jeppesen \\ Research Center COM, Technical University of Denmark, Building 345V, DK-2800 Kongens Lyngby, Denmark, \\ mg@com.dtu.dk \\ H.-J. Deyerl*, N. Plougmann**, M. Kristensen*** \\ *Physics Department, Technical University of Chemnitz,Germany, **Research Department, Rigshospitalet, Denmark, ***Department of \\ Physics and Astronomy, University of Aarhus, Denmark,
}

\begin{abstract}
Raman-assisted wavelength conversion for ultra-high speed data is characterised in two approaches: a novel scheme based on cross-phase modulation using specially designed notch filters and a $160 \mathrm{~Gb} / \mathrm{s}$ experiment based on self-phase modulation.

(C) 2004 Optical Society of America

OCIS codes: (060.4370) Nonlinear optics, fibers; (060.4510) Optical communications
\end{abstract}

\section{Introduction}

Methods for wavelength conversion at high bit rates $(160 \mathrm{~Gb} / \mathrm{s}$ and above) will be essential tools in a possible future implementation of high-speed optical communication systems. A limited number of wavelength conversion schemes have been devised, including [1-4]. Recently, Raman gain has been shown to aid the conversion process in schemes utilising non-linear processes in highly non-linear fibres, schemes such as Cross-Phase Modulation (XPM) of a continuous wave (CW) light beam [1] and Self-Phase Modulation (SPM) of the data itself [2].

This paper investigates challenges in high-speed operation of these wavelength conversion schemes. In the XPM approach of [1], the generated sideband of the CW is filtered out with a broad bandpass filter, which will limit the optical signal to noise ratio, as it is difficult to suppress the CW beam this way. Here we introduce a strong notch filter for the CW suppression, and we show that it is possible to convert pulses suitable for $160 \mathrm{~Gb} / \mathrm{s}$. Furthermore, error free wavelength conversion using the Raman-assisted SPM scheme is demonstrated with a 160 $\mathrm{Gb} / \mathrm{s}$ data signal multiplexed from a $40 \mathrm{~Gb} / \mathrm{s}$ base rate.

\section{XPM for wavelength conversion}

One way of achieving high speed wavelength conversion is using the XPM product between high power data pulses and a CW probe signal at separate wavelengths. The XPM process will broaden the spectrum of the CW where a data mark is co-propagating, whereas the CW will experience no XPM when no data mark is co-propagating. In this way a wavelength converted data signal can be extracted as the XPM broadening of the CW. This can be done in practice by suppressing the original data signal and the CW using spectral filtering.

The set-up for XPM-based wavelength conversion is shown on the figure below.

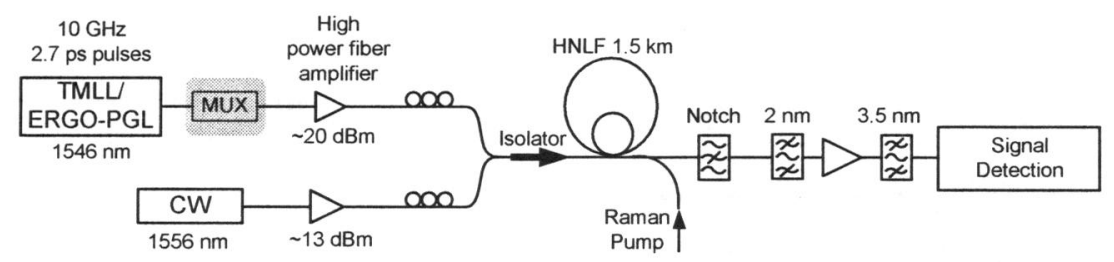

Fig. 1 Set-up for XPM wavelength conversion

XPM is performed between a train of short pulses (2.7 ps for a Tuneable Mode-Locked Laser (TMLL) and 2.2 ps for an ERbium Glass Oscillator Pulse Generating Laser (ERGO-PGL)) and a CW probe. The $10 \mathrm{GHz}$ pulse train can be multiplexed to higher bit rates using a passive fibre delay multiplexer. The two signals are amplified and combined before injecting them into $1.5 \mathrm{~km}$ of HNLF $\left(\gamma \sim 10 \mathrm{~W}^{-1} \mathrm{~km}^{-1}, \lambda_{0} \sim 1552 \mathrm{~nm}, \mathrm{~S} \sim 0.017 \mathrm{ps} / \mathrm{nm}^{2} \mathrm{~km}\right)$. To amplify the non-linear process, the HNLF is Raman pumped $(200 \mathrm{~mW})$ in a counter-propagating configuration. The spectrum of the signals exiting the HNLF is filtered using a notch filter to suppress the CW wavelength and a bandpass filter to suppress the original $10 \mathrm{GHz}$ pulse train as well as part of the XPM product. The use of a notch filter to suppress the CW is advantageous at high bit rates as it relaxes the requirements of the bandpass filter characteristics, since at high bit rates a broad spectral range of the XPM product must be extracted to avoid pulse broadening, and at the same time the CW should be strongly suppressed. 


\section{CMQ2}

\section{Pulse properties}

The product of XPM between a data mark and the CW is seen in the spectral domain as sidebands forming on either side of the CW. It is these sidebands which can be extracted as the wavelength converted data by filtering. The relation between the scaling of the two XPM sidebands in the wavelength conversion output and the suppression of pedestal levels in the pulse autocorrelations is revealed in fig. 2.

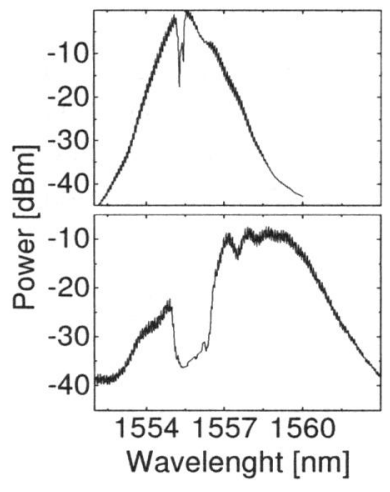

(a)

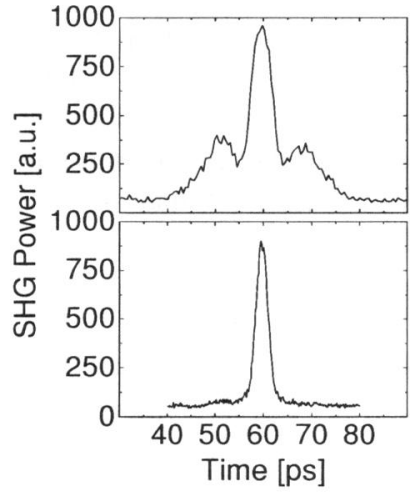

(b)

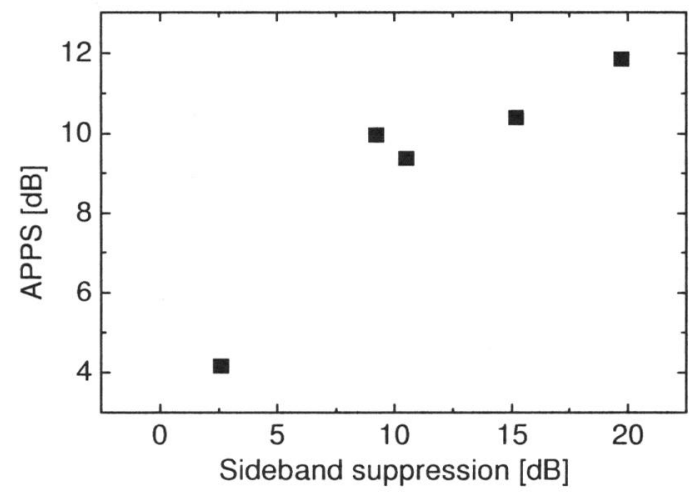

(c)

Fig. 2 Spectra and autocorrelations for converted pulses (a) and (b). Results for Autocorrelation Pulse Pedestal Suppression (APPS) dependence on sideband suppression are shown in (c)

When both XPM sidebands are transmitted, i.e. at low sideband suppression (fig. 2a upper), the autocorrelation pulse shape is deteriorated by high pedestals (fig. $2 \mathrm{~b}$ upper). In the case shown a notch filter with $\sim 0.3 \mathrm{~nm}$ bandwidth and $\sim 25 \mathrm{~dB}$ suppression is used to suppress the $\mathrm{CW}$. Adjusting the bandpass filter to increasingly suppress one sideband increases the pulse pedestal suppression. The case of strong sideband suppression and the following autocorrelation pulse pedestal suppression (APPS) is seen in the lower graphs of fig. 2a and 2b. Here a notch filter with $2 \mathrm{~nm}$ bandwidth and $\sim 77 \mathrm{~dB}$ suppression is used to suppress the $\mathrm{CW}$ and the central portion of the lower wavelength sideband. The observations are summarised in the plotted measurements of fig. 2c. At stronger sideband suppression no pulse pedestals are discernable on the linear autocorrelator that is used for these measurements, and so the tendency of increasing APPS continues beyond the measurements of fig. $2 \mathrm{c}$.

The dependence of the pedestals on the sideband suppression is due to a phase shift between the two XPM induced sidebands. This results in interference between the two spectrally separated pulses when only the CW is suppressed. Suppressing one sideband minimises this pulse splitting effect and pulses suitable for high-speed operation are obtained, i.e. pedestal-free and 2.1 ps FWHM (fig. 2b) and low amplitude noise (fig. 3b).

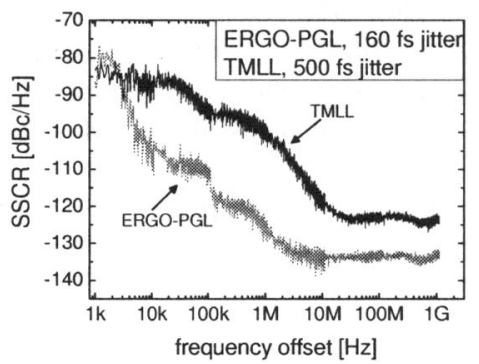

(a)

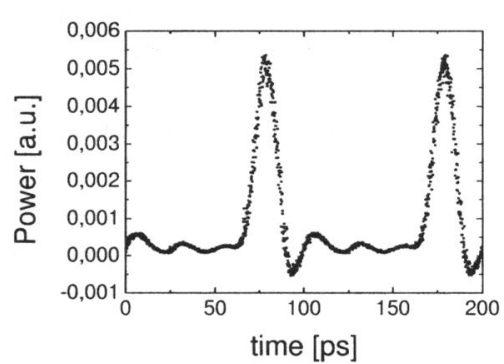

(b)

Fig. 3 (a) Single Sideband to Carrier Ratio (SSCR) measured for two pulse sources. Jitter was determined according to the ITU recommendation of integrating from $20 \mathrm{kHz}$ to $80 \mathrm{MHz}$. (b) Wavelength converted pulses with low amplitude noise using the TMLL pulse source.

A strong dependence between the properties of the pulse source and the quality of the converted signal is observed. Using a TMLL with a high phase noise level (timing jitter $500 \mathrm{fs}$ ), fig. 3a, as $10 \mathrm{GHz}$ pulse source for conversion, a converted signal with low amplitude noise is obtained, fig. 3b. Alternatively, using an ERGO-PGL with timing jitter $\sim 160 \mathrm{fs}$, fig. 3a, as pulse source, results in a significant amount of amplitude noise being added to the converted signal. A similar tendency is seen using this source for SPM, fig. 4c. The added noise is believed to stem from Stimulated Brillouin Scattering (SBS) induced by the very narrow line width of the ERGO-PGL, which has considerably less phase noise than the TMLL used for these experiments. SBS is inversely proportional to the line 


\section{CMQ2}

2005 Conference on Lasers \& Electro-Optics (CLEO)

width and causes amplitude noise through transfer of optical power from the forward propagating signal to the backscattered SBS product. As the phase noise adds to the line width, the TMLL with more timing jitter actually helps to reduce the amplitude noise. So there is a compromise between phase and amplitude noise. The CW light has its line width increased using the built-in coherence control of the laser source.

\section{SPM based wavelength conversion}

An alternate scheme for wavelength conversion is spectral broadening by SPM. The setup for this scheme is simpler than the one used for XPM since no CW is inserted into the HNLF and no notch filter is used for filtering the output. The data signal is multiplexed from a $40 \mathrm{~Gb} / \mathrm{s}$ base rate to $160 \mathrm{~Gb} / \mathrm{s}$ and amplified to $\sim 30 \mathrm{dBm}$. SPM then causes strong spectral broadening of the data pulses and wavelength conversion can be performed by selecting a new spectral range using bandpass filtering at the output of the HNLF. Raman pumping of the HNLF has been seen to significantly improve the SPM wavelength conversion [2].

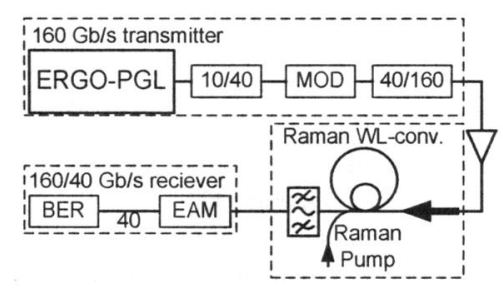

(a)

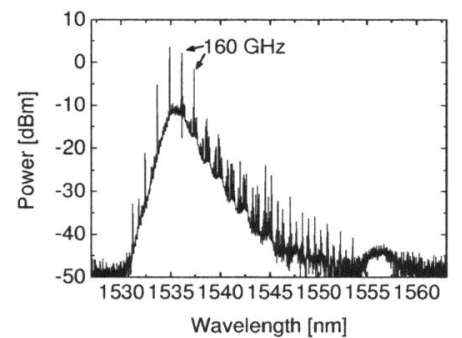

(b)

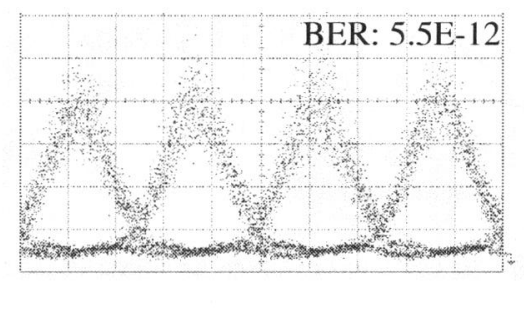

(c)

Fig. $4160 \mathrm{~Gb} / \mathrm{s}$ SPM wavelength converted signal. (a) Experimental set-up for SPM wavelength conversion. (b) The spectrum of the wavelength converted signal using $1 \mathrm{~km}$ of HNLF with $\lambda_{0} \sim 1559 \mathrm{~nm}$. (c) Clear and open eye diagram for the error free converted and demultiplexed signal.

In fig. $4 \mathrm{a}$ is shown the set-up for $160 \mathrm{~Gb} / \mathrm{s}$ SPM wavelength conversion using the low-jitter ERGO-PGL pulse source and an ElectroAbsorption Modulator (EAM) as demultiplexer. Fig. 4b shows the spectrum of the converted signal. The eye diagram shown in fig. $4 \mathrm{c}$ for the converted signal has clear and open eyes but also some amplitude noise. The system provides error free operation with a bit error rate of $5.5^{*} 10^{-12}$ with a Raman pump power of $\sim 700$ $\mathrm{mW}$ - without Raman error free operation was not possible with this set-up, which is in agreement with the observations in [2]. In this setup the wavelength of both the original and the converted data is placed in the negative dispersion region to minimise noise from higher order soliton effects. A significant amount of amplitude noise is observed on the demultiplexed signal due to SBS.

\section{Conclusion}

This paper has investigated aspects of the XPM and SPM schemes for high-speed wavelength conversion. Using notch filters for CW suppression in the XPM conversion approach is shown to allow conversion of narrow pulses suitable for $160 \mathrm{~Gb} / \mathrm{s}$ operation. The relation between sideband suppression and autocorrelation pulse pedestals is characterised. Error free wavelength conversion of a $160 \mathrm{~Gb} / \mathrm{s}$ data signal using the SPM scheme with Ramanassistance is demonstrated.

It is believed that the observations and relationships reported in this paper can aid in optimising the performance of wavelength converters based on the XPM- and SPM-schemes.

\section{References}

[1] W. Wang, et. al., “All-Optical Label Switching/Swapping of 160 Gbps Variable Length packets and 10 Gbps Labels using a WDM Raman Enhanced-XPM Fiber Wavelength Converter with Unicast/Multicast Operation," in Proc. of OFC, PDP8 (2004).

[2] M. Galili, et. al., "160 Gb/s Raman-Assisted SPM Wavelength Converter," in Proc. of ECOC, Th4.3.1, (2004)

[3] S. Watanabe et. al., "160 Gb/s Optical 3R-Regenerator in Fiber Transmission experiment," in Proc. of OFC, PD16-1 (2003)

[4] S. Nakamura et. al., "168 Gb/s all-optical wavelength conversion with a symmetric-Mach-Zender-type switch," IEEE Photon. Technol. Lett. 13(10), $1091(2001)$ 